\title{
Understanding the building blocks of the paediatric dentistry curriculum for undergraduate students in an Australian University
}

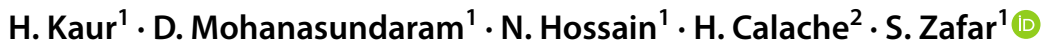

Received: 29 July 2021 / Accepted: 14 December 2021 / Published online: 29 January 2022

(c) The Author(s) under exclusive licence to European Academy of Paediatric Dentistry 2021

\begin{abstract}
Purpose To investigate the attitude of final-year dental students towards their paediatric dentistry training and their confidence in treating child patients.

Methods A 55-item questionnaire was distributed online and physically to the final-year BDSc (Hons) students at The University of Queensland (Australia). The questionnaire consisted of four parts including theoretical knowledge, clinical observational experience, preclinical training and clinical training in paediatric dentistry. Self-reported confidence was recorded using five-point Likert scale questions ranging from 'not confident at all' to 'completely confident', which were allocated the numbers 1-5, respectively. Jamovi and GraphPad Prism were used for data analysis and creation of graphs.

Results A total of 47 students completed the questionnaire giving a response rate of $77 \%$. Approximately two-thirds of participants had previous experience working with children and $70 \%$ had observed a practitioner providing paediatric dental treatment. The students reported the lowest level of clinical confidence for pulp therapy $(M=2.32 ; S D=1.08)$. The clinical confidence in dental trauma management was also reported to be low $(M=2.50 ; S D=1.15)$. The clinical administration of local anaesthetic (LA) had the highest level of confidence $(M=3.95 ; S D=1.03)$. The students reported that they were highly confident in the theoretical knowledge of behaviour guidance techniques $(M=3.64 ; S D=0.97)$ and preclinical training involving examination, treatment planning and preventative procedures $(M=4.33, S D=0.67)$.

Conclusion This study showed that students reported low levels of confidence in pulp therapies and trauma management in children as compared to other aspects of paediatric dentistry. Students indicated the need for more preclinical and clinical training sessions, as well as more opportunities to perform a wider variety of treatments on paediatric patients.
\end{abstract}

Keywords Confidence $\cdot$ Curriculum $\cdot$ Dental education $\cdot$ Paediatric dentistry $\cdot$ Students $\cdot$ Undergraduate

\section{Introduction}

Paediatric dentistry is defined as "the branch of dentistry that is concerned with preventative and therapeutic oral health care for children from birth through to adolescence and those with special needs" (Dental Board of Australia 2016). The oral health of children and adolescents is critical in determining oral health outcomes later on in life as poor oral health in childhood is strongly associated with poor

S. Zafar

s.zafar@uq.edu.au

1 School of Dentistry, The University of Queensland, Brisbane, 288 Herston Road, Herston, Brisbane, QLD 4006, Australia

2 Department of Dentistry and Oral Health, La Trobe Rural Health School, La Trobe University, Melbourne, Australia oral health in adulthood (Australian Institute of Health and Welfare 2020). Hence, the provision of effective paediatric dental treatment can help prevent poor oral health throughout the patient's lifetime.

The non-fatal burden of oral disease among Australian children is quite significant, accounting for $8.1 \%$ and $4.1 \%$ of the non-fatal burden of disease in children aged 5-9 and 10-14, respectively (Australian Bureau of Statistics 2016). Furthermore, the National Child Oral Health Survey (NCOHS) conducted in Australia found 27\% and 11\% of children aged 5-14 years had untreated dental caries in their primary and permanent dentitions, respectively (Do and Spencer 2016). Moreover, the rate of potentially preventable hospitalisations (PPHs) of children aged $0-14$ years in 2017-2018 due to dental conditions was 17 per 1000 population (Australian Institute of Health and Welfare 2020). This high prevalence of oral diseases poses a significant burden 
on Australian children and their families, thus creating a significant treatment burden on dental practitioners (Batista et al. 2011). There are only 151 specialist paediatric dentists across Australia, meaning there is a ratio of approximately one specialist for every 42,500 paediatric patients (Australian Bureau of Statistics 2017; Dental Board of Australia 2017). There is clearly an inadequate specialist workforce to manage the high number of paediatric patients. Consequently, it is crucial for general dentists and oral health therapists to have the skills and confidence to manage the routine dental treatment needs of Australian children. Thus, it is important that the paediatric dentistry curricula provided at Australian universities for general dentists and oral health therapists ensure that graduates possess the necessary knowledge, skills and attitudes to provide effective routine dental care for paediatric patients and, as a result, reduce the overall burden of oral disease among Australian children.

Treating paediatric patients can be stressful for both the oral health practitioner and the child. The failure to provide a positive dental experience to younger patients may lead to dental fear and avoidance persisting for the rest of their lives (Kan et al. 2019). Previous literature has highlighted that, although behaviour guidance is a key aspect in the provision of healthcare to paediatric patients, it is not given adequate importance in healthcare training (Batista et al. 2011). Furthermore, evidence shows that even when the majority of dental students felt that they had received adequate anxiety management teaching, they still lacked confidence in managing anxiety in their paediatric patients (Pine and McGoldrick 2000). A lack of confidence in managing patient behaviour translates to stress in dental students which can negatively affect their future paediatric dental practice (Cardoso et al. 2004).

Existing international literature has identified that the aspect of paediatric dentistry in which students are the least confident is the management of dental trauma (Rodd et al. 2010; Sonbol et al. 2017; Walley et al. 2014). A 2010 study conducted at three UK dental schools (Liverpool, Manchester and Sheffield), investigated the self-reported clinical confidence in paediatric dentistry (Rodd et al. 2010). Confidence was reported to be the lowest in the management of dental trauma, which is of concern as paediatric patients may experience many financial, psychological and dental complications due to poor trauma management (Rodd et al. 2010). Based on the conclusions of the aforementioned study, the paediatric dentistry curricula at these schools were modified to include seven trauma case scenarios and timetabling changes were implemented at the Liverpool Dental School to increase clinical exposure to trauma cases (Rodd et al. 2010; Walley et al. 2014). The survey was re-conducted in the same dental schools after three years and dental trauma management still had the lowest level of student confidence (Walley et al. 2014).
Walley et al. conceded that the presentation of trauma cases to student clinics is unpredictable and so, it is impossible to ensure equitable exposure which may explain why student confidence was still lacking (Walley et al. 2014). A similar study conducted at the University of Jordan Dental School also found that students were the least confident in the management of dental trauma (Sonbol et al. 2017).

Students in the UK have been reported to be the most confident in providing preventative treatments (including oral hygiene instruction, dietary counselling, topical application of fluoride and fissure sealants) to a child patient (Rodd et al. 2010; Walley et al. 2014). They also felt well prepared to provide routine paediatric dental care after graduation (Rodd et al. 2010; Walley et al. 2014). Students in Jordan felt the most confident in providing preventative treatments as well as examination, diagnosis, treatment planning and performing operative dentistry (Sonbol et al. 2017). However, there is no such study exploring self-reported confidence in Australian dental students, thus giving no indication of the confidence level of newly graduated dental professionals in various aspects of paediatric dentistry.

The building blocks of paediatric dentistry curriculum for the Bachelor of Dental Science (Honours) at The University of Queensland (Australia) consist of four components: theoretical, observational, preclinical and clinical. Dental students undergo theoretical learning (lectures, tutorials, case studies and e-learning) in their third and fourth year of study. In their fourth year, students attend preclinical sessions where they are trained in fissure sealants, ultraconservative restorations, pulpotomies, preformed metal crowns (PMCs) and strip crowns. In the same year, students attend the Oral Health Centre (OHC) paediatric clinic to observe (approximately $3 \mathrm{~h}$ per week) a final-year student treating paediatric patients. The $\mathrm{OHC}$ clinics, which are run through the Oral Health Alliance between The University of Queensland and MetroNorth Hospital and Health Services, provide dental services to public patients (School of Dentistry 2021). At the $\mathrm{OHC}$ paediatric clinic, students are supervised by paediatric dental specialists. Students undergo clinical placements in their fifth and final year of study, half of which are completed at the $\mathrm{OHC}$, where they spend one day per week at the $\mathrm{OHC}$ paediatric clinic (equally split between treating paediatric patients and assisting colleagues providing treatment). For the other half of the placements, students are placed at an external public dental clinic which does not have dedicated paediatric dentistry sessions. This curriculum is designed to ensure dental students obtain the expected knowledge and skills in paediatric dentistry to meet the certifying criteria of the Australian Dental Council (2016). Such training would allow students to improve their confidence in treating paediatric patients. The aim of this pilot study was to obtain an understanding of the attitudes of final-year dental 
students towards their paediatric dentistry training and to explore how their training has affected their self-reported confidence in various aspects of paediatric dentistry.

\section{Methods}

This cross-sectional study used a 55-item questionnaire to investigate the students' perception of the paediatric dentistry training gained at The University of Queensland. Ethical approval was obtained through the Human Research Ethics Committee (Approval No. ID: 2020001747).

\section{Study participants}

Participants were recruited from the 2020 cohort of finalyear undergraduate dental students. The inclusion criteria were final-year dental students who agreed to participate in the study through either completing the questionnaire via hard-copy or online formats. The online questionnaire was generated using the online survey tool, Qualtrics ${ }^{\mathrm{XM}}$ (Qualtrics, Provo, Utah, USA). The participants were provided with the information sheet and written consent was obtained.

\section{Survey tool}

The questionnaire was developed to assess the self-reported attitudes and confidence of dental students towards various aspects of paediatric dentistry. It was constructed by the researchers and piloted with ten individuals in the dental field (including students, dentists and paediatric specialists) before implementation and was modified according to the feedback received. The questionnaire consisted of two sections. Section A recorded the demographics of the sample population. Section B comprised questions on various aspects of paediatric dentistry divided into four components including theoretical knowledge, observation clinics, preclinical training and clinical training. The majority of the multiple choice questions were presented through five-point Likert scale questions, comprising of either 'strongly disagree' to 'strongly agree' or 'not confident at all' to 'completely confident'. The multiple choice questions were based on building blocks of paediatric dentistry-related activities, as used in the study by Rodd et al. (2010). These building blocks of paediatric dentistry were modified to reflect the paediatric dentistry curriculum taught at The University of Queensland and included key concepts from the Handbook of Paediatric Dentistry as endorsed by the Australasian Academy of Paediatric Dentistry (Cameron and Widmer 2013). Hence, the questions in Section B were categorised under the following core areas; (1) Examination, treatment planning and preventative treatment, (2) Behaviour guidance, (3) Local anaesthesia (LA) administration, (4)
Restorative procedures, (5) Dental trauma management, (6) Extractions and (7) Pulp therapies.

Online data collection was carried out between September 2020 and November 2020. Two months after the initial distribution of the online questionnaire, two reminder emails were sent to encourage more responses from participants. In November 2020, hard-copy questionnaires were distributed at the $\mathrm{OHC}$ to students who reported they had not completed the online questionnaire. Questionnaires were collected by researchers after completion.

\section{Statistical analysis}

The data were tabulated on Microsoft Excel spreadsheet (Version 2008) and then imported into Jamovi (Version 1.6.3) Statistics for Windows (Microsoft, Redmond, Washington, USA) for descriptive analysis and GraphPad PRISM 9.0 software (GraphPad Software, San Diego, Calif., USA) for collation and creation of appropriate graphs. Responses were summarised and comparisons were made using Student's $t$ test. The responses for the Likert scale questions, which go from 'strongly disagree' through to 'strongly agree' or 'not confident at all' to 'completely confident', were allocated the numbers $1-5$ in that respective order. The results were expressed as descriptive statistics, such as counts and percentages. Results of the Likert Scale questions were expressed as mean \pm standard deviation $(S D)$ from 1 (strongly disagree/not confident at all) to 5 (strongly agree/ completely confident).

\section{Results}

\section{Demographic information}

All undergraduate final-year dental students $(n=61)$ were invited to participate in this study and 47 completed the questionnaire; hence, the response rate was $77 \%$. Majority of students (80.9\%) were between 20 and 25 years old. Out of the 47 participants, $22(46.8 \%)$ respondents were female and $24(51.5 \%)$ were male. One participant preferred not to specify their gender. Almost two-thirds of participants (63.8\%) had previous work experience with children mainly in the form of volunteering, babysitting and paid work as dental assistants (Table 1).

\section{Theoretical knowledge}

Students were most confident with the theoretical knowledge of behaviour guidance techniques $(M=3.64 ; S D=0.97)$ but were least confident with pulp therapy content $(M=2.95$; $S D=0.89$ ). No data about the theoretical content regarding extractions in a child patient were collected (Fig. 1). 
Table 1 Demographics of participants

\begin{tabular}{ll}
\hline Characteristic & $n(\%)$ \\
\hline Age & \\
$<20$ & $1(2.1)$ \\
$20-25$ & $38(80.9)$ \\
$25-30$ & $6(12.8)$ \\
$30-35$ & $2(4.3)$ \\
$>35$ & $0(0)$ \\
Gender & \\
Female & $22(46.8)$ \\
Male & $24(51.1)$ \\
Prefer not to say & $1(2.1)$ \\
Previous work experience with \\
children \\
Yes \\
No & $30(63.8)$ \\
\hline
\end{tabular}

Students reported that the most confidence with theoretical content was developed through traditional in-person lectures $(M=3.61 ; S D=0.81)$ followed by case studies $(M=3.50$; $S D=0.88$ ). E-learning (videos, virtual patients and online workshops) was the delivery format which was reported as 'neutral' in developing student confidence $(M=2.98$; $S D=1.19$ ) (Table 2).

\section{Paediatric dentistry clinical observations}

Responses indicated that $33(70.2 \%)$ of students had shadowed a clinician performing dental treatment on a paediatric patient. Table 3 provides information on where these observations were held, the qualification of the observed practitioners and the procedure observed. A total of $20(60.7 \%)$ respondents out of 33 somewhat agreed/ strongly agreed that clinical observations helped them gain
Table 2 Student confidence developed from various formats of theoretical content delivery

\begin{tabular}{lcc}
\hline Theoretical content delivery format & Mean & SD \\
\hline Lectures & 3.61 & 0.81 \\
Tutorials & 3.25 & 1.10 \\
Case studies & 3.50 & 0.88 \\
Self-study & 3.36 & 0.99 \\
E-learning & 2.98 & 1.19 \\
\hline
\end{tabular}

confidence in managing children's behaviour during dental treatment and $69.7 \%$ agreed that these observations helped them gain confidence in a dentist's ethical responsibilities when treating paediatric dental patients. After undertaking paediatric observations, $69.7 \%$ of respondents agreed that they felt more prepared and confident in treating paediatric patients (Table 4). Nearly half $(45.5 \%)$ of the participants were interested in spending more time in these observational clinics.

\section{Preclinical training}

Students developed the most confidence in examination, treatment planning and preventative procedures from their preclinical training $(M=4.33 ; S D=0.67)$. Behaviour guidance techniques, LA administration and dental trauma management did not have a preclinical teaching component. Despite receiving preclinical training in pulp therapies, participants felt least confident $(M=2.56$; $S D=1.10)$ in this aspect and felt the need for additional sessions (Fig. 1). In fact, students requested more preclinical sessions to practice all aspects of paediatric dentistry. Overall, $71.1 \%$ of students reported that they felt 'fairly'
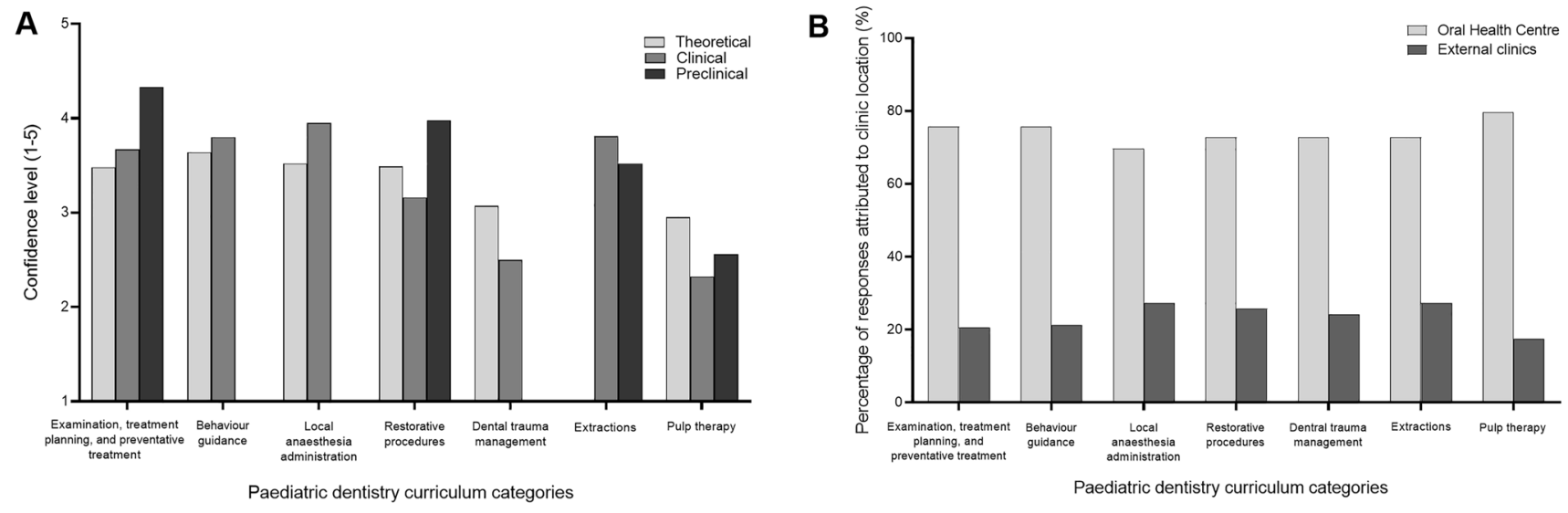

Fig. 1 A Student confidence levels in paediatric dentistry related to theoretical learning, preclinical training and clinical training. B Confidence gained in paediatric dental treatment from placement at the oral health centre and external clinics 
Table 3 Observation experiences of participants

\begin{tabular}{ll}
\hline Observation characteristic & $n(\%)$ \\
\hline Observation Experience & $33(70.2)$ \\
Yes & \\
Where was the observation held & $4(12.1)$ \\
General dental clinic & $4(12.1)$ \\
Paediatric dental clinic & $24(72.7)$ \\
Oral Health Centre & $4(12.1)$ \\
Public dental clinic & $9(27.3)$ \\
Private dental clinic & $0(0)$ \\
Other & \\
Qualification of observed practitioner & $18(54.5)$ \\
General dental practitioner & $16(48.5)$ \\
Paediatric dental specialist & $20(60.6)$ \\
5th-year dental students at the Oral Health Centre & $1(3.0)$ \\
Other & \\
Procedure observed & $25(75.8)$ \\
Fissure sealants & $24(72.7)$ \\
Restorations & $9(27.3)$ \\
Preformed metal crowns & $4(12.1)$ \\
Strip crowns & $20(60.0)$ \\
Extractions & $4(12.1)$ \\
Pulp therapies & $6(18.2)$ \\
Orthodontics & $22(66.7)$ \\
Behaviour management techniques &
\end{tabular}

and 'completely' confident in their manual dexterity skills developed from paediatric preclinical sessions.

\section{Clinical training}

Participants were most confident in the clinical administration of LA to a paediatric patient $(M=3.95 ; S D=1.03)$. Contrary to this, participants felt least confident in performing pulp therapy procedures on a child patient $(M=2.32$; $S D=1.08$ ) (Fig. 1A). An open-ended question that asked the students whether there were any clinical procedures that they would have liked more preclinical training on, revealed that approximately $70 \%$ of respondents would have liked more preclinical training for pulp therapy procedures. Additionally, the clinical management of dental trauma also had a poor confidence level $(M=2.50 ; S D=1.15)$. Confidence in clinical paediatric dental procedures was developed predominantly through placements at the OHC rather than through placements at external clinics (Fig. 1B), and the difference was statistically significant $(p<0.001)$. More than threequarters $(73.1 \%)$ of students surveyed agreed that the teaching style of their clinical supervisors (ie. paediatric dental specialists) boosted their confidence in treating paediatric patients. Half of the participants agreed with the statement, "I feel I have received sufficient clinical paediatric dentistry experience". Conversely, $26.2 \%$ of students disagreed and $23.8 \%$ were neutral. The students who believed they had not received sufficient experience suggested improvements to their clinical training in the form of more clinical time and the opportunity to perform a wider variety of treatments on children.

\section{Discussion}

This study, which is the first of its kind undertaken in Australia, aimed to explore the confidence and attitudes of finalyear dental students in various aspects of paediatric dentistry. The theory related to behaviour guidance techniques had the highest level of confidence which was likely developed through a blended-learning approach involving in-person lectures with adjunctive online case studies. This topic was taught to students via lectures and then students had to complete a role-playing video assessment item where they managed an uncooperative child patient (played by either the students themselves or a younger sibling) under various scenarios. The video allowed students to apply the theory in a simulated clinical environment which would have strengthened student confidence. Such blended-learning strategies could also be applied in the theoretical learning of pulp therapies which students were least confident in. The theoretical content for pulp therapies was only delivered as lecture.

Table 4 Student confidence gained after observing dental treatment on paediatric patients

\begin{tabular}{|c|c|c|c|c|c|}
\hline \multirow[t]{2}{*}{ Statements } & \multicolumn{5}{|l|}{ Responses $n(\%)$} \\
\hline & Strongly disagree & $\begin{array}{l}\text { Some- } \\
\text { what } \\
\text { disagree }\end{array}$ & Neutral & Somewhat agree & Strongly agree \\
\hline $\begin{array}{l}\text { The observations helped me gain confidence in behaviour guidance } \\
\text { techniques of child management }\end{array}$ & $3(9.1)$ & $1(3.0)$ & $9(27.3)$ & $15(45.5)$ & $5(15.2)$ \\
\hline $\begin{array}{l}\text { The observations helped my confidence in my ethical responsibilities } \\
\text { when treating children }\end{array}$ & $3(9.1)$ & $1(3.0)$ & $6(18.2)$ & $18(54.5)$ & $5(15.2)$ \\
\hline $\begin{array}{l}\text { After observations clinics, I feel more prepared/ confident in treating } \\
\text { paediatric patients }\end{array}$ & $3(9.1)$ & $2(6.1)$ & $5(15.2)$ & $18(54.5)$ & $5(15.2)$ \\
\hline
\end{tabular}


However, and addition of adjunct online components, training using dental simulators and additional hands-on practice in simulation laboratory could be embedded into the paediatric dentistry curriculum to enhance students' experience. A recent cross-sectional study compared students' perception of the preclinical training for pulpotomies and PMCs using dental simulators. The study concluded that dental simulators could be used as an adjunct for paediatric dentistry preclinical training (Zafar et al. 2020). Furthermore, a review conducted by Ruiz et al. (2006) concluded that online learning should not replace traditional teaching but should complement it. Therefore, students could develop confidence in pulp therapies by applying theoretical concepts in a simulated environment to manage an online virtual patient.

Pulp therapies were the least observed procedures by students during their observation rotations in fourth year. Observational learning, where the learner collaborates with a more experienced learner, has been shown to improve the student's knowledge retention, problem-solving abilities and motivation (Mills and Bernstein 2021). The lack of observational learning for pulp therapies may therefore have negatively influenced student confidence levels. Confidence in complex clinical procedures, such as pulp therapies and dental trauma management, could be improved by providing students with wider observational opportunities as part of their external placements. It is the authors' opinion that the inclusion of student observations of paediatric dental specialists providing treatment under relative analgesia and general anaesthesia would be beneficial as these modalities allow for the provision of more complex treatment that would not be possible in a non-compliant child patient under LA. Wider paediatric dentistry observational opportunities as such would improve student confidence in paediatric dentistry.

Students had a mean confidence score between fairly and completely confident for their preclinical training in the examination, treatment planning and preventative treatment for a child patient. In Australia, The Child Dental Benefits Schedule (CDBS) is a Medicare funded program providing AUD 1000 worth of dental treatments every two years for eligible children (2-17 years). CDBS covers a range of services, including examinations, cleaning, radiographs, restorations, fissure sealants, extractions and root canal therapy. The CDBS does not cover orthodontics or cosmetic dental treatment (Services Australia, Australian Government 2019). In 2020, approximately $85 \%$ of the services provided under the CDBS were diagnostic and preventative (Services Australia, Australian Government 2020). Students had high levels of confidence in these areas of paediatric dentistry and so the curriculum does appear to be adequately developing student confidence to provide a large proportion of routine care to a child patient. However, students were not confident in their preclinical practice of pulp therapies. A large proportion of students wanted more preclinical sessions for pulp therapy practice but Barakat et al. (2021) found that additional preclinical training in root canal treatment (in the permanent dentition) did not significantly improve confidence. Students currently receive only one three-hour preclinical session to practice a pulpotomy on a deciduous tooth. Hence, preclinical training could be broadened to include more sessions on pulp therapies. Further research is required to determine whether additional preclinical sessions would increase student confidence in paediatric dentistry.

The clinical administration of LA in a child had the highest level of confidence. This is in contrast to Batista et al. (2011) who reported lower levels of student confidence when performing invasive and potentially aversive procedures such as LA administration in children. Students developed confidence with this procedure as the theory was taught to students in their second year and applied in a preclinical setting at the same time. However, this learning was not tailored towards paediatric patients and so the curriculum was modified after this cohort of students was surveyed for this study. The modification to the curriculum included the use of a virtual reality simulator as an adjunct to teaching (Zafar et al. 2021). Strong practitioner confidence in LA administration is essential in preventing dental fear and anxiety in children.

Clinical confidence levels for the management of dental trauma and pulp therapies were below neutral. These findings of low confidence in carrying out primary molar pulp therapies and dental trauma management have been replicated in multiple studies from the UK and Jordan (Rodd et al. 2010; Sonbol et al. 2017; Walley et al. 2014). Additionally, this study demonstrated that pulp therapies were the clinical aspect of paediatric dentistry that students were least confident in. This may be due to lack of clinical experience as students stated they "have not done enough pulpotomies or pulpectomies in young children" and there was a "lack of complex cases that require pulp therapy as they are treated under general anaesthesia." A quarter of the students disagreed that they had received enough clinical paediatric dentistry experience and stated that their clinical experience could have been improved with the opportunity to perform a wider variety of treatments on child patients. Another reason could be that the trends towards pulpotomy in children are generally decreasing due to the availability and popularity of minimal intervention dentistry including indirect pulp therapy, hall technique, silver diamine fluoride and selective caries removal.

The provision of equitable opportunity for students to apply their teaching clinically is an important factor for the development of student confidence. Rodd et al. (2010) recognised that it is a challenge for universities to provide equitable opportunity to treat complex cases especially for dental trauma as the presentation of children with traumatic 
injuries is unpredictable. Possible solutions include triaging cases requiring specific treatments to students who have not experienced those treatments. Also, students reported that clinical placements at $\mathrm{OHC}$ were integral in the development of student confidence in trauma management and pulp therapies and therefore should continue to be included in the curriculum. Dedicating sessions to treat paediatric patients at external placement locations (under the supervision of a paediatric dental specialist) would be beneficial as communitybased placements have been shown to increase student confidence due to more exposure to pulp therapy procedures and extractions (Coe et al. 2018). Increasing clinical experience in certain dental procedures should translate to increased student confidence in performing those procedures.

A limitation of this study is that students' clinical experience was not recorded. The questionnaire measured selfreported confidence associated with paediatric clinical procedures which could have been reduced due to poor confidence in theoretical content and preclinical training or due to a lack of clinical experience. As the questionnaire did not record the latter, it is difficult to determine what impacted the confidence levels of students in the clinical practice of paediatric dentistry. Clinical experience and its direct effect on confidence need to be investigated further in future studies by either auditing clinical placement logbooks and recording treatment provided or by asking students about their clinical experience in the administered questionnaire.

Finally, the findings of this study cannot be generalised since the standard clinical experience of students treating paediatric patients was reduced due to the COVID-19 pandemic. Students lost $28 \mathrm{~h}$ of paediatric clinical practice due to lockdown restrictions in their final year of study. This study should be repeated in a future cohort, who are unaffected by lockdown restrictions, to evaluate their confidence levels following the standard number of clinical hours during placement.

\section{Conclusion}

Considering the limitations of the present study, moderate to high confidence levels in all core areas of paediatric dentistry except pulp therapies and dental trauma management were shown. Pulp therapies were also found to be the least observed procedure. Students indicated the need for more preclinical and clinical training sessions, as well as more opportunities to perform a wider variety of treatments on paediatric patients.

Supplementary Information The online version contains supplementary material available at https://doi.org/10.1007/s40368-021-00688-1.
Acknowledgements The authors would like to acknowledge the specialist paediatric dentists who piloted the questionnaire including Emeritus Professor Kim Seow, Dr. Yvonne Lai, Dr. William Fogarty, Dr. Victoria Kashchuk, and Dr. Sonali Mistry. We would also like to acknowledge the students who participated in this study.

Author contributions Conceptualisation: SZ, HC; Methodology: NH, HK, DM; Formal analysis and investigation: NH, HK, DM; Writingoriginal draft preparation: $\mathrm{NH}, \mathrm{HK}, \mathrm{DM}$; Writing — review and editing: SZ, HC; Supervision: HC, SZ.

Funding The authors did not receive financial support from any organisation for the submitted work.

Availability of data and material The data used for this research article can be made available upon request to the authors.

Code availability The online questionnaire was generated and distributed using Qualtrics ${ }^{\mathrm{XM}}$ (Qualtrics, Provo, Utah, USA). Microsoft Excel (Version 2008) was used to tabulate the data and then imported into Jamovi (Version 1.6.3) Statistics for Windows (Microsoft, Redmond, Washington, USA) for descriptive analysis. GraphPad PRISM 9.0 software (GraphPad Software, San Diego, Calif., USA) was used for the production of graphs.

\section{Declarations}

Conflict of interest The authors declare they have no financial conflict of interests related to this study. Author SZ is the Discipline Lead for Paediatric Dentistry for The University of Queensland's School of Dentistry. Author NH, HK, and DM are BDSc (Hons) students at The University of Queensland's School of Dentistry.

Ethics approval The questionnaire and methodology for this study were approved by the Human Research Ethics committee of the University of Queensland (Ethics approval number: 2020001747).

Consent to participate Informed consent was obtained from all individual participants included in the study.

\section{References}

Australian Bureau of Statistics. 2016 Census QuickStats. Canberra: Australian Bureau of Statistics. In: Census 2017. https://quick stats.censusdata.abs.gov.au/census_services/getproduct/census/ 2016/quickstat/036. Accessed: Mar 2020.

Australian Dental Council. Professional competencies of the newly qualified dentist. In: Program accreditation. 2016. https://www. adc.org.au/Program-Accreditation/Professional-Competencies. Accessed Jul 2021.

Australian Institute of Health and Wellfare. Dental and oral health. In: Health conditions, disability \& deaths. 2020. https://www.aihw. gov.au/reports-data/health-conditions-disability-deaths/dentaloral-health/about. Accessed Jun 2021.

Barakat RM, Matoug-Elwerfelli M, Almohareb RA, Balto HA. Influence of preclinical training on root canal treatment technical quality and confidence level of undergraduate dental students. Int J Dent. 2021;2021:9920280. https://doi.org/10.1155/2021/9920280.

Batista CG, Nascimento CL, Rolim GS, Rocha RA, Rodrigues AF, Ambrosano GM, et al. Student self-confidence in coping with uncooperative behaviours in paediatric dentistry. Eur J Dent Educ. 
2011;15(4):199-204. https://doi.org/10.1111/j.1600-0579.2010. 00656.x.

Cameron AC, Widmer RP. A Handbook of Pediatric Dentistry. 4th ed. Edinburgh: Mosby. 2013.

Cardoso CL, Loureiro SR, Nelson-Filho P. Pediatric dental treatment: manifestations of stress in patients, mothers and dental school students. Braz Oral Res. 2004;18(2):150-5. https://doi.org/10. 1590/s1806-83242004000200011.

Coe JM, Brickhouse TH, Bhatti BA, Best AM. Impact of communitybased clinical training on dental students' confidence in treating pediatric patients. J Dent Educ. 2018;82(1):5-11. https://doi.org/ 10.21815/JDE.018.002.

Dental Board of Australia. Dental list of recognised specialties, related specialist titles and definitions. In: Registration standards. 2017. https://www.dentalboard.gov.au/registration-standards.aspx. Accessed Jun 2021.

Do LG, Spencer AJ. Oral health of Australian children: the National Child Oral Health Study 2012-14. Adelaide: University of Adelaide Press; 2016.

Kan S, Ho V, Siddiqi A, Zafar S. The prevalence of percutaneous exposure incidents among staff and students treating pediatric patients. J Dent Child (chic). 2019;86(2):81-7.

Mills DA, Bernstein J. A community of practice in dental education: a phenomenon of newcomers becoming oldtimers. J Dent Educ. 2021;85(7):1259-66. https://doi.org/10.1002/jdd.12582.

Pine CM, McGoldrick PM. Application of behavioural sciences teaching by UK dental undergraduates. Eur J Dent Educ. 2000;4(2):4956. https://doi.org/10.1034/j.1600-0579.2000.040201.x.

Rodd HD, Farman M, Albadri S, Mackie IC. Undergraduate experience and self-assessed confidence in paediatric dentistry: comparison of three UK dental schools. Br Dent J. 2010;208(5):221-5. https:// doi.org/10.1038/sj.bdj.2010.207.

Ruiz JG, Mintzer MJ, Leipzig RM. The impact of E-learning in medical education. Acad Med. 2006;81(3):207-12. https://doi.org/10. 1097/00001888-200603000-00002.

School of Dentistry. Oral Health Alliance. In: About Oral Health Alliance. 2021. https://dentistry.uq.edu.au/about/oral-health-alliance. Accessed Jul 2021.
Services Australia. Australian Government. Child Dental Benefits Schedule. In: Medicare payments and services. 2019. https://www. servicesaustralia.gov.au/individuals/services/medicare/child-dental-benefits-schedule?. Accessed Jul 2021.

Services Australia. Australian Government. Requested MBS category by group and subgroup processed from January 2020 to December 2020. In: Medicare Group Reports. 2021. http://medicarestatistics. humanservices.gov.au/statistics/do.jsp?_PROGRAM=\%2Fstatisti cs\%2Fmbs_group_standard_report $\& D R I L L=$ on $\&$ GROUP $=\mathrm{A} \&$ $\mathrm{VAR}=$ services $\&$ STAT $=$ count $\&$ RPT_FMT $=$ by + state $\&$ PTYPE $=$ calyear\&START_DT $=202001 \& E N D \_D T=202012$. Accessed Jun 2021.

Sonbol HN, Abu-Ghazaleh SB, Al-Bitar ZB. Undergraduate experience and self-assessed confidence in paediatric dentistry at the University of Jordan Dental School. Eur J Dent Educ. 2017;21(4):e12630. https://doi.org/10.1111/eje.12233.

Walley S, Bailey JR, Albadri S, Mackie IC, Gilchrist F, Rodd HD. Undergraduates' self-reported clinical experience, confidence and perspectives of hospital and outreach paediatric dentistry: a three-year multi-centre evaluation. Br Dent J. 2014;216(5):251-6. https://doi.org/10.1038/sj.bdj.2014.144.

Zafar S, Lai Y, Sexton C, Siddiqi A. Virtual Reality as a novel educational tool in pre-clinical paediatric dentistry training: students' perceptions. Int J Paediatr Dent. 2020;30(6):791-7. https://doi. org/10.1111/ipd.12648.

Zafar S, Siddiqi A, Yasir M, Zachar JJ. Pedagogical development in local anaesthetic training in paediatric dentistry using virtual reality simulator. Eur Arch Paediatr Dent. 2021. https://doi.org/10. 1007/s40368-021-00604-7.

Publisher's Note Springer Nature remains neutral with regard to jurisdictional claims in published maps and institutional affiliations. 